\title{
Calculation of field enhancement factor and screening effects in carbon nanotube arrays
}

\author{
$\underline{\text { R. C. Smith }}{ }^{*}$, L.D. Filip, J. D. Carey and S. R. P. Silva \\ Nano-Electronics Centre, Advanced Technology Institute, University of Surrey, Guildford, GU2
}

$7 X H, U K$.

Computational 3D simulations of individual carbon nanotubes (CNTs) and vertically aligned arrays of CNTs were conducted in order to gain insight into their field emission characteristics. We find that the widely used approximation that the enhancement factor is equal to the aspect ratio of the emitter to be an over-estimation, and that the enhancement factor is only loosely related to the aspect ratio, among other parameters.

In order to derive a theoretical model for enhancement factor of an isolated CNT, we show that we can model a CNT as a series of stacked spheres. Using this approach we apply a modified induced charge model to accurately predict the local field at the tip of the apex of the CNT. Figure 1 shows the enhancement factor for a range of aspect ratios of CNT. It is observed that simulating the CNT as a series of conductive spheres does not differ in terms of output from the standard hemispherical cap on a cylinder model, as with the output associated with our model based on a superposition of potentials on each sphere in the series, and using an image charge method for calculating the local field at the apex of the CNT. Also, we see a good agreement to our model.

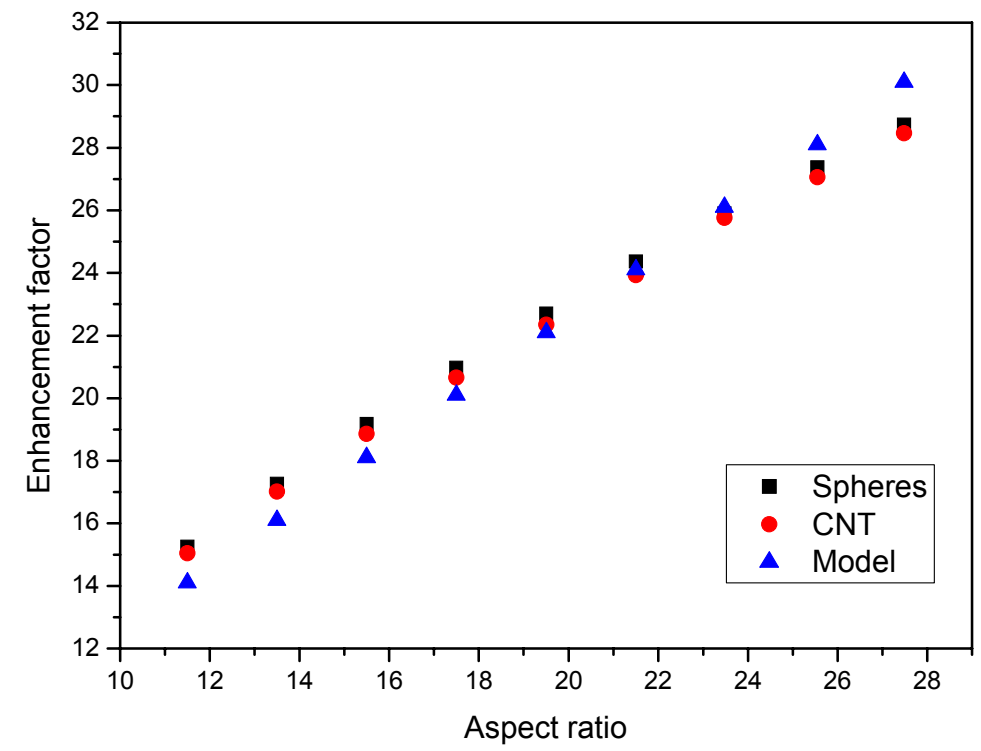

Fig. 1. Simulated enhancement factors for CNT (O), stacks of spheres $(\square)$, and corresponding values calculated by our model $(\Delta)$. 
We also present current work relating to the field screening effect of arrays of CNTs. In this work we show that the approximation that the inter-tube separation has to be twice the height of the CNT, greatly underestimates the effects of screening. An inter-tube separation of 5 times the height of the CNT is shown to reduce the effects of screening to a minimum. At a distance of two times the CNT height, a residual screening of the order of $11 \%$ remains within the array. We were able to replicate the widely used approximation of inter-tube separation of $S=2 h$, based on both experimental and simulation data performed in the past, when we simulated a line of CNT, mimicking a two dimensional setup.

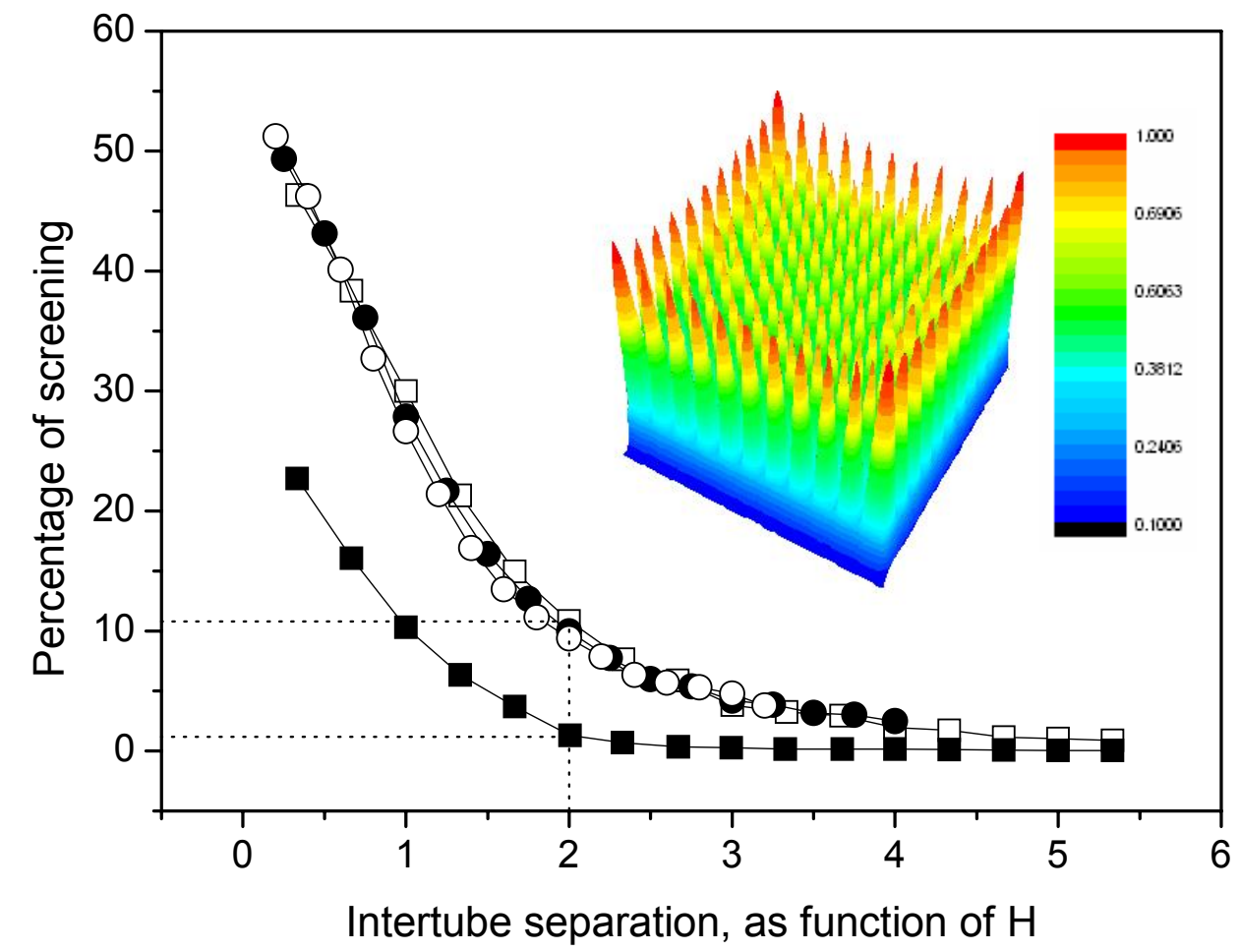

Percentage of screening calculated by the difference in local electric field between an isolated CNT, to the middle CNT of an array of 9 . Constant radius of $50 \mathrm{~nm}$ was used, and CNT height of $3(\square), 4(\odot)$ and $5 \mu \mathrm{m}(O)$ is shown. At $S=2 h$, the middle CNT is screened by approximately $11 \%$. For comparison, 3 CNT each spaced by $3 \mu \mathrm{m}$ was simulated in 2D (ם). Here it is seen that at $S=2 h$ the screening factor is less than $2 \%$. Inset shows the local field for an array of 11 by 11 CNT.

To analyse the effects seen in this theoretical study, we were able to comment on the three dimensional aspect of field screening, and draw comments as to the implication of these effects when designing large area ordered arrays of CNT for vacuum microelectronic applications.

* Email: r.c.smith@eim.surrey.ac.uk 